\title{
The 2013 ACC/AHA risk score and subclinical cardiac remodeling and dysfunction: Complementary in cardiovascular disease prediction
}

\author{
Nicholas Cauwenberghs ${ }^{\text {a }}$, Kristofer Hedman ${ }^{\text {b, c }}$, Yukari Kobayashi ${ }^{\mathrm{b}}$, Thomas Vanassche ${ }^{\mathrm{d}}$, \\ Francois Haddad ${ }^{\text {b }}$, Tatiana Kuznetsova ${ }^{\text {a, * }}$ \\ ${ }^{a}$ Research Unit Hypertension and Cardiovascular Epidemiology, KU Leuven Department of Cardiovascular Sciences, University of Leuven, Belgium \\ b Stanford Cardiovascular Institute, Department of Medicine, Stanford University, California, USA \\ c Department of Clinical Physiology, and Department of Medical and Health Sciences, Linköping University, Sweden \\ ${ }^{\mathrm{d}}$ Centre for Molecular and Vascular Biology, KU Leuven Department of Cardiovascular Sciences, University of Leuven, Belgium
}

\section{A R T I C L E I N F O}

\section{Article history:}

Received 15 July 2019

Received in revised form

16 September 2019

Accepted 20 September 2019

Available online $\mathrm{xxx}$

\section{Keywords:}

Cardiovascular risk score

Echocardiography

Atherosclerotic cardiovascular disease

Risk stratification

\begin{abstract}
A B S T R A C T
Background: Echocardiography might enhance cardiovascular (CV) risk stratification beyond tools grading the risk for atherosclerotic CV diseases (ASCVD). We therefore studied the complementarity between the ASCVD risk score recommended by American cardiology societies and echocardiographic profiling in predicting adverse CV outcome in the community.

Methods: 984 community-dwelling individuals between 40 and 79 years old (51.3\% women) underwent CV risk profiling and echocardiography. We estimated their 10-year ASCVD risk from baseline risk factors using the Pooled Cohort Equations. Participants were categorized as at low $(<2.5 \%)$, borderline (2.5$<7.5 \%)$ or intermediate-to-high $(\geq 7.5 \%)$ ASCVD risk. Main outcome was the incidence of CV events collected on average 7.5 years later.

Results: The probability for cardiac remodeling and/or dysfunction as assessed by echocardiography rose progressively with increasing 10-year ASCVD risk. During follow-up, 116 participants experienced at least one CV endpoint (15.8 events per 1000 person-years). With increasing 10-year ASCVD risk, the CV event rate increased stronger in participants with $\geq 1 \mathrm{LV}$ abnormality at baseline. Indeed, in individuals with an intermediate-to-high ASCVD risk and $\geq 1 \mathrm{LV}$ abnormality at baseline, the risk was significantly higher than the average population risk for a first CV event (HR: 3.00, $\mathrm{P}<0.001$ ). Adding the presence of $\geq 1 \mathrm{LV}$ abnormality to a ASCVD risk score-based model yielded significant improvement in C-statistics $(P=0.024)$, integrated discrimination $(P=0.0085)$ and net reclassification $(P<0.001)$ for adverse CV events.

Conclusions: Echocardiographic profiling enhanced CV risk stratification in individuals at intermediateto-high ASCVD risk. Echocardiographic screening might supplement traditional ASCVD risk grading for $\mathrm{CV}$ disease prediction.
\end{abstract}

() 2019 Elsevier B.V. All rights reserved.

\section{Statement of authorship}

All authors take responsibility for all aspects of the reliability and freedom from bias of the data presented and their discussed interpretation.

\section{Sources of funding}

The Research Unit Hypertension and Cardiovascular Epidemiology receives grants from the Fonds voor Wetenschappelijk Onderzoek Vlaanderen, Brussels, Belgium (grants 11Z0916N and G0C5319N).

\section{Conflict of interest}

* Corresponding author.

E-mail address: tatiana.kouznetsova@kuleuven.be (T. Kuznetsova).
None. 


\section{Introduction}

Cardiovascular (CV) diseases pose a major burden on public health and healthcare [1,2]. The prevalence of CV diseases remains high as a result of increased life expectancy and risk factors such as hypertension and diabetes mellitus. Of note, in the presence of CV risk factors, the heart gradually remodels $[3,4]$ and its function progressively declines [5,6] years to decades before symptoms present. Recent heart failure guidelines already emphasized the need to adequately detect subclinical phases of cardiac maladaptation and remodeling in order to modify risk factors and timely counter CV pathophysiology [7].

$\mathrm{CV}$ risk scores play a central role in tailoring $\mathrm{CV}$ preventive strategies. In 2013, the American College of Cardiology (ACC) and the American Heart Association (AHA) derived sex- and racespecific estimates from 4 population cohorts to calculate the 10year risk for a first atherosclerotic CV disease (ASCVD) in individuals 40 to 79 years of age [8]. The resulting Pooled Cohort Equations were found to outperform other risk models such as SCORE and the Framingham Risk Score in predicting CV disease $[9,10]$. Recent CV prevention guidelines now recommend the use of the Pooled Cohort Equations to assess 10-year ASCVD risk and to start a clinician-patient discussion to decide on the type and intensity of $\mathrm{CV}$ preventive measures such as initiation of statin therapy [9,11-14].

Risk enhancers such as biochemical and imaging biomarkers might provide incremental information beyond the 2013 ACC/AHA risk score. Therefore, the ACC and the AHA advocate to investigate the incremental value of nontraditional risk enhancers beyond the Pooled Cohort Equations for ASCVD risk prediction [8]. Within this context, detection of subclinical maladaptation and malfunctioning of the heart by echocardiography might augment CV disease prediction of the currently endorsed risk grading. Previously, echocardiographic abnormalities reflecting subclinical yet adverse left ventricular (LV) remodeling $[4,15]$ and systolic $[16,17]$ and diastolic dysfunction [18] were found to independently of traditional risk factors predict CV outcome in the community. Moreover, a combination of echocardiographic remodeling and dysfunction indexes proved complementary for CV outcome in asymptomatic subjects [16]. So far, the additive value of LV hypertrophy for CV disease prediction has been demonstrated beyond non-endorsed CV risk scores based on traditional risk factors [19,20].

To date, however, no study has yet investigated the potential incremental value of echocardiographic profiles for CV outcome prediction beyond ASCVD risk assessment recommended by cardiology societies. In this population study, we therefore explored for the first time the complementarity between ASCVD risk score and echocardiographic profiling in predicting adverse CV outcome.

\section{Methods}

\subsection{Study population}

The Flemish Study on Environment, Genes and Health Outcomes (FLEMENGHO), a large family-based population resource on the genetic epidemiology of cardiovascular phenotypes, received a priori approval from the Ethics Committee of the University of Leuven. We randomly recruited a population sample in northern Belgium [21]. Seven Belgian municipalities provided listings of all inhabitants sorted by address. Households were the sampling unit. We numbered households and generated a random-number list using SAS software (SAS Institute, Cary, NC). Households with a number matching the list were invited; household members older than 18 years were eligible. For this particular sub-study, we invited 1851 individuals for an examination including echocardiography from May 2005 to January 2015. We obtained written informed consent in 1447 participants (participation rate, 78.2\%). As the Pooled Cohort Equations apply to individuals between 40 and 79 years only, we excluded 381 participants either younger than 40 $(n=341)$ or older than 79 years old $(n=40)$. We additionally excluded 82 participants presenting atrial fibrillation $(n=7)$, a cardiac pacemaker $(n=5)$ or suboptimal echocardiographic image quality $(\mathrm{n}=70)$. In total, we statistically analyzed 984 subjects (see Supplemental Figure 1 for study flow chart).

\subsection{Echocardiographic profiling}

Using a Vivid 7 Pro or Vivid E9 (GE Vingmed, Horten, Norway), two experienced observers obtained conventional echocardiographic images along the parasternal and apical axes [16,22].

One observer (T.K.) performed conventional echocardiographic measurements blinded to the participants' characteristics using EchoPac software version 113 (GE Vingmed, Horten, Norway) as recommended [22] and as detailed in Supplemental Methods. LV concentric remodeling was defined as a relative wall thickness $($ RWT $)>0.42$ [22]. LV hypertrophy was an LV mass of $50 \mathrm{~g} / \mathrm{m}^{2.7}$ in men and $47 \mathrm{~g} / \mathrm{m}^{2.7}$ in women $[22,23]$. We considered participants with an $\mathrm{E} / \mathrm{e}^{\prime}$ ratio (a non-invasive surrogate of $\mathrm{LV}$ filling pressure) $>8.5$ as having LV diastolic dysfunction [18]. Diastolic dysfunction was confirmed using differences in durations between mitral A flow and reverse pulmonary veins flow, tricuspid regurgitation and elevation in left atrial volume index [18]. Two experienced observers (T.K. and N.C.) derived LV longitudinal strain (LS) using myocardial speckle-tracking software (Q-analysis, GE Vingmed) [24]. An absolute global LS below $17.4 \%$ in men and $18.5 \%$ in women was suggestive of early LV systolic dysfunction. The thresholds used to define the echocardiographic abnormalities were previously shown to predict cardiac events in the community $[16,18,23]$.

\subsection{CV risk profiling}

We administered a standardized questionnaire to collect information on the subject's medical history, smoking and drinking habits and medication intake. We verified and supplemented selfreported disease by medical records provided by general practitioners and regional hospitals. Brachial blood pressure was the average of 5 auscultatory readings obtained in seated position. Fasting blood samples were drawn for measurement of serum creatinine, total cholesterol, HDL-C and blood glucose. Definitions of hypertension, diabetes mellitus and renal failure are specified in Supplemental Methods.

\subsection{Assessment of 10-year ASCVD risk}

We applied the sex-specific Pooled Cohort Equations for white Caucasians to estimate the 10 -year risk for a first ASCVD event as endorsed by the 2013 ACC/AHA Guideline on the Assessment of Cardiovascular Risk [8]. These equations enclose age, sex, systolic blood pressure, antihypertensive treatment, total cholesterol, HDLC, current smoking status, history of diabetes mellitus and interactions between these risk factors [8]. Based on the risk score distribution (Supplemental Figure 2), we categorized participants without a prior $\mathrm{CV}$ event as at low $(<2.5 \%, \mathrm{n}=292)$, borderline (2.5-7.5\%, $\mathrm{n}=289)$ or intermediate-to-high $\mathrm{CV}$ risk $(\geq 7.5 \%$, $\mathrm{n}=300)$. In the 2018 ACC/AHA lipid guidelines, the $7.5 \%$ limit represents the cut-off for eligibility for statin treatment in primary prevention [12]. Participants who had experienced a CV event before the baseline examination $(n=103)$ were assigned to a separate group (very high risk). 47, 31 and 25 subjects respectively 
experienced 1, 2 or more CV events before the baseline examination. Supplemental Table 1 lists the cause-specific CV morbidity of these participants at the time of the examination.

\subsection{Assessment of outcome}

To study the incidence of CV events in relation to baseline ACC/ AHA risk score and subclinical echocardiographic abnormalities, we collected outcome data on average $7.5 \pm 3$.6 years after the baseline examination. We ascertained the vital status of the participants until January 17, 2017. During follow-up, 73 participants died. We applied the International Classification of Disease codes for the immediate and underlying cause of death [16]. We assessed the incidence of non-fatal events via a follow-up visit or a telephone interview, repeating the standardized questionnaire used at baseline. All self-reported diseases were ascertained and supplemented by medical records provided by general practitioners and regional hospitals [16]. Fatal and non-fatal CV events comprised coronary events, heart failure, atrial fibrillation, life-threatening arrhythmias, pulmonary hypertension, stroke, transient ischemic attack, aortic aneurysm, arterial embolism and arterial revascularization. Only the first event was considered in outcome analyses. Supplemental Table 2 lists the cause-specific incidence of CV mortality and morbidity during follow-up.

\subsection{Statistical analysis}

We used SAS version 9.4 (SAS Institute, Cary, NC, USA) for database management and statistical analysis. Means and proportions were compared by a large sample $z$-test and $\chi^{2}$ test, respectively. Level of significance was set at a 2 -sided $P<0.05$. We evaluated the distributions of all variables and normalized them by logarithmic transformation if needed. We used the Kaplan-Meier method for estimation of the $\mathrm{CV}$ event free survival according to the ASCVD risk profile and the presence of LV abnormalities (LV remodeling, abnormal LS and/or diastolic dysfunction). Excluding subjects with previous CV events, we also calculated Cox regression hazard ratios for CV events per ASCVD/LV profile subgroup, expressing the hazard ratio versus the average risk of the 881 subjects at primary $\mathrm{CV}$ risk. Finally, we assessed the improvement in $\mathrm{CV}$ event prediction when adding the echocardiographic features to the ACC/AHA risk score by evaluating the improvements in Cstatistic, integrated discrimination (IDI) and continuous net reclassification (NRI) indexes [25].

\section{Results}

\subsection{Characteristics of participants}

The mean age of the 984 participants ( $52.3 \%$ women, $51.5 \%$ hypertensive) was 57.0 (SD, 10.1) years. Table 1 lists the clinical and echocardiographic characteristics per CV risk group. As expected, participants at intermediate-to-high ASCVD risk were significantly older and had a higher prevalence of hypertension, diabetes mellitus and renal failure than the $<7.5 \%$ risk groups $(P<0.001$; Table 1). Left atrial volume, RWT, LV mass, and E/e' ratio were significantly higher whereas E/A ratio and e' peak were significantly lower in participants at intermediate-to-high risk than in those with a low or borderline 2013 ACC/AHA risk score $(P<0.001$; Table 1).

Of note, the prevalence of hypertension, diabetes mellitus and renal failure did not differ between the group with intermediateto-high 2013 ACC/AHA risk score and the patients with previous CV events $(P \geq 0.050$; Table 1$)$. In contrast, left atrial volume and LV mass indexes and $E / e^{\prime}$ ratio were significantly higher in the $C V$ patients than in the participants at intermediate-to-high ASCVD risk $(P \leq 0.0073$; Table 1$)$.

\section{2. $L V$ remodeling and dysfunction and the 2013 ACC/AHA risk score}

The prevalence of LV remodeling, an abnormal LS and diastolic dysfunction was respectively $32.9 \%, 23.5 \%$ and $11.4 \%$ in the 881 participants without previous CV events and $66.0 \%, 39.9 \%$ and $47.6 \%$ in the $103 \mathrm{CV}$ patients (Fig. 1A). The prevalence of LV remodeling and dysfunction increased progressively from low to intermediate/ high 10-year ASCVD risk (Fig. 1B and C). Indeed, participants at intermediate-to-high ASCVD risk and those with a previous CV event had significantly higher odds to present LV concentric remodeling (OR, 4.84 and 4.30), LV hypertrophy (OR, 5.93 and 12.3), abnormal LS (OR, 2.04 and 3.05) and LV diastolic dysfunction (OR, 25.3 and 65.3) than participants at low risk $(P<0.001$ for all; Supplemental Figure 3). In support, the probability for maladaptive LV phenotypes rose progressively with the 10-year ASCVD risk increasing on a continuous scale (Supplemental Figure 4).

\subsection{Prediction of incident $C V$ events by 10-year ascvd risk and echocardiographic profiles}

The median follow-up time was 7.8 years ( 5 th to 95th percentile, 2.3-12.3). During 7334 person-years of follow-up, 116 participants experienced at least one fatal or nonfatal CV endpoint (15.8 events per 1000 person-years). Supplemental Figure 5 presents the CV event rates per 1000 person-years by ASCVD risk quintiles and LV profiles. With increasing 10-year ASCVD risk, the CV event rate increased stronger in participants with at least one LV abnormality at baseline (Supplemental Figure 5).

Fig. 2 shows the CV event-free survival by combinations of 10year ASCVD risk ( $<7.5 \% \mathrm{vs} \geq 7.5 \%)$ and the presence or absence of $\geq 1 \mathrm{LV}$ abnormality. In subjects with a 10 -year ASCVD risk above $7.5 \%$, the incidence of $\mathrm{CV}$ events increased significantly if at least one LV abnormality was present at baseline (Fig. 2A). In contrast, the presence of an LV abnormality at baseline did not discriminate the incidence of $C V$ events in subjects with a 10-year ASCVD risk below 7.5\% (Fig. 2A). In support, compared to the average population risk of subjects free from $\mathrm{CV}$ events at baseline, only those who had a 10 -year ASCVD risk $\geq 7.5 \%$ and $\geq 1 \mathrm{LV}$ abnormality at baseline presented a higher risk for a first CV event during follow-up (HR: 3.00; 95\% CI, 2.13-4.23, $P<0.001$ ) (Fig. 2B). Supplemental Figure 6 provides Cox HRs for CV events associated with 10-year ASCVD risk ( $<7.5 \%$ vs $\geq 7.5 \%$ ) and each cardiac maladaptive profile (normal LV versus concentric remodeling, hypertrophy, abnormal LS and diastolic dysfunction).

For prediction of adverse CV events, C-statistic increased with 0.029 (95\% CI: 0.004 to $0.053 ; P=0.024$ ), relative IDI was $14.6 \%$ $(P=0.0085)$ and continuous NRI valued $0.54(95 \% \mathrm{CI}: 0.33$ to 0.76 ; $P<0.001)$ when adding the presence of at least $1 \mathrm{LV}$ abnormality to an ASCVD risk score-based model (Table 2). Adding the presence of $\geq 1$ LV abnormality to an ASCVD risk score-based model yielded significant improvement in C-statistics $(0.029 ; P=0.024)$, integrated discrimination $(14.6 \% ; P=0.0085)$ and net reclassification $(0.54 ; P<0.001)$ indexes for adverse CV events. When adding the LV features separately to an ASCVD risk score-based model, particularly inclusion of LV diastolic dysfunction improved the prognostic accuracy for CV events, given the significant increase in C-statistic (0.026), the relative IDI (23.9\%) and the NRI (0.46) $(P \leq 0.016$ for all) (Table 2$)$. 
Table 1

Clinical and echocardiographic characteristics of 984 FLEMENGHO participants.

\begin{tabular}{|c|c|c|c|c|}
\hline \multirow[b]{2}{*}{ Characteristic } & \multicolumn{3}{|c|}{ 10-year ASCVD risk } & \multirow[t]{2}{*}{ Previous CV event $(n=103)$} \\
\hline & $<2.5 \%(\mathrm{n}=292)$ & $2.5-7.5 \%(n=289)$ & $\geq 7.5 \%(\mathrm{n}=300)$ & \\
\hline \multicolumn{5}{|l|}{ Anthropometrics } \\
\hline Age, years & $47.6(4.9)$ & $54.4(6.3)^{*}$ & $64.7(7.6)^{*, \dagger}$ & $68.6(7.4)^{*, \dagger, \ddagger}$ \\
\hline Female sex, No. (\%) & $228(78.1)$ & $131(45.3)^{*}$ & $108(36.0)^{*, \dagger}$ & $38(36.9)^{*}$ \\
\hline Body mass index, $\mathrm{kg} / \mathrm{m}^{2}$ & $25.6(4.2)$ & $27.1(4.1)^{*}$ & $27.6(3.9)^{*}$ & $26.8(3.5)^{*}$ \\
\hline Systolic BP, mmHg & $122.7(12.8)$ & $129.9(13.5)^{*}$ & $141.5(16.6)^{*, \dagger}$ & $143.3(19.3)^{*, \dagger}$ \\
\hline Diastolic BP, mmHg & $80.5(8.7)$ & $83.2(8.6)^{*}$ & $84.1(9.8)^{*}$ & $79.1(9.2)^{t, \ddagger}$ \\
\hline Heart rate, bpm & $60.9(9.6)$ & $61.1(8.7)$ & $60.8(9.9)$ & $56.5(8.9)^{*, \dagger, \ddagger}$ \\
\hline \multicolumn{5}{|l|}{ Biochemical data } \\
\hline Serum creatinine, $\mu \mathrm{mol} / \mathrm{L}$ & $74.9(14.6)$ & $79.1(13.3)^{*}$ & $83.4(15.5)^{*, \dagger}$ & $90.0(27.0)^{*, \dagger, \ddagger}$ \\
\hline eGFR, $\mathrm{ml} / \mathrm{min}$ per $1.73 \mathrm{~m}^{2}$ & $84.6(15.8)$ & $85.0(16.8)^{*}$ & $80.2(17.9)^{*, \dagger}$ & $74.6(19.2)^{*, \dagger, t}$ \\
\hline Total cholesterol, mg/dL & $198.7(35.5)$ & $206.6(33.5)^{*}$ & $208.0(37.3)^{*}$ & $182.2(32.9)^{*, \dagger, \ddagger}$ \\
\hline $\mathrm{HDL}, \mathrm{mg} / \mathrm{dL}$ & $63.0(15.6)$ & $55.3(13.1)^{*}$ & $53.3(14.3)^{*}$ & $52.3(13.7)^{*, \dagger}$ \\
\hline Blood glucose, $\mathrm{mmol} / \mathrm{L}$ & $4.69(0.40)$ & $4.84(0.70)^{*}$ & $5.00(0.84)^{*, \mathrm{~b}}$ & $5.11(1.29)^{*, \dagger}$ \\
\hline \multicolumn{5}{|l|}{ Questionnaire and clinical data } \\
\hline Current smoking, No. (\%) & $30(10.3)$ & $64(22.2)^{*}$ & $71(23.7)^{*}$ & $13(12.6)^{\dagger, \ddagger}$ \\
\hline Drinking alcohol, No. (\%) & $104(35.6)$ & $124(42.9)^{*}$ & $124(41.3)$ & $35(34.0)$ \\
\hline Hypertensive, No. (\%) & $69(23.6)$ & $130(45.0)^{*}$ & $222(74.0)^{*, \dagger}$ & $86(83.5)^{*, \dagger}$ \\
\hline Treated for hypertension, No. (\%) & $27(9.3)$ & $68(25.5)^{*}$ & $128(42.7)^{*, \dagger}$ & $72(69.9)^{*,+, \ddagger}$ \\
\hline On lipid-lowering drugs, No. (\%) & $26(8.9)$ & $43(14.9)^{*}$ & $66(22.0)^{*, \dagger}$ & $49(47.6)^{*, \dagger, \ddagger}$ \\
\hline History of diabetes, No. (\%) & $5(1.7)$ & $5(1.7)$ & $31(10.3)^{*, \dagger}$ & $12(11.7)^{*, \dagger}$ \\
\hline Renal failure, No. (\%) & $10(3.4)$ & $9(3.1)$ & $25(8.3)^{*, \dagger}$ & $22(21.4)^{*, \dagger}$ \\
\hline \multicolumn{5}{|l|}{ Echocardiographic data } \\
\hline LA volume index, $\mathrm{ml} / \mathrm{m}^{2}$ & $29.4(6.9)$ & $30.9(8.3)^{*}$ & $33.7(10.0)^{*, \dagger}$ & $38.6(12.8)^{*, \dagger, \ddagger}$ \\
\hline LV relative wall thickness & $0.35(0.048)$ & $0.38(0.055)^{*}$ & $0.40(0.062)^{*, \dagger}$ & $0.40(0.063)^{*, \dagger}$ \\
\hline LV mass/body height ${ }^{2.7}, \mathrm{~g} / \mathrm{m}^{2.7}$ & $37.1(8.3)$ & $41.9(8.8)^{*}$ & $46.5(10.6)^{*, \dagger}$ & $52.2(14.9)^{*, \dagger, \ddagger}$ \\
\hline LV ejection fraction, \% & $62.0(5.8)$ & $61.2(5.8)$ & $60.6(5.7)^{*}$ & $59.1(7.4)^{*, \dagger}$ \\
\hline LV global LS, \% & $19.8(1.9)$ & $19.2(2.1)^{*}$ & $19.0(2.4)^{*}$ & $18.4(2.9)^{*, \dagger}$ \\
\hline E/A ratio & $1.39(0.38)$ & $1.14(0.30)^{*}$ & $0.94(0.25)^{*, \dagger}$ & $0.95(0.35)^{*, \dagger}$ \\
\hline $\mathrm{e}^{\prime}$ peak, $\mathrm{cm} / \mathrm{s}$ & $12.1(2.3)$ & $10.2(2.2)^{*}$ & $8.36(2.06)^{*, \dagger}$ & $7.40(2.20)^{*, \dagger, \ddagger}$ \\
\hline E/e' ratio & $6.59(1.47)$ & $7.10(1.72)^{*}$ & $8.22(2.40)^{*, \dagger}$ & $9.39(4.15)^{*, \dagger, \pm}$ \\
\hline
\end{tabular}

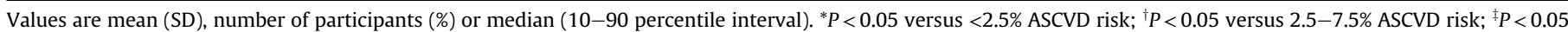

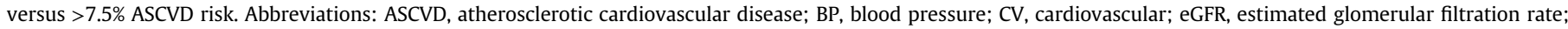
HDL, high-density lipoprotein; LA, left atrium; LS, longitudinal strain; LV, left ventricular.

\section{Discussion}

In this community-based study, we explored the complementarity between the 2013 ACC/AHA risk grading and echocardiographic profiling in predicting CV outcome. We observed that: i) in subjects at intermediate-to-high ASCVD risk ( $\geq 7.5 \%)$, the incidence of adverse CV events increased significantly if at least one LV abnormality was present at baseline; and that ii) addition of echocardiographic features to the 2013 ACC/AHA risk score improved the prognostic accuracy for predicting future CV outcome.

Previous observational studies reported associations between the 2013 ACC/AHA risk score and other subclinical CV organ damage such as silent brain infarctions [26] and the presence [27] and progression of coronary artery calcification [28]. Other communitybased studies observed that the risk for LV hypertrophy increased with greater overall CV risk [29,30]. To our knowledge, our population study is the first to report associations between an ASCVD risk score and subclinical cardiac abnormalities. Indeed, we observed that the likelihood for subclinical heart remodeling and both systolic and diastolic dysfunction rose significantly with increasing 10-year ASCVD risk.

The 2013 ACC/AHA risk score endorsed by American cardiology societies seemed to be able to identify individuals at high risk for subclinical cardiac maladaptation and malfunctioning, particularly for early LV diastolic dysfunction. Myocardial ischemia as induced by an atherosclerotic disease such as coronary artery disease (CAD) slows ventricular relaxation, impairs ventricular distensibility and, in consequence, can trigger diastolic dysfunction [31]. In support of our findings, in 2042 participants of the Olmsted County study, subclinical LV diastolic dysfunction was detected in $57.7 \%$ of the participants with a history of CAD and only in $24.7 \%$ of the participants free from CAD [32]. Of note, metabolites of fatty acid oxidation and inflammation were upregulated in CAD patients with advanced stage of diastolic dysfunction [33], highlighting metabolic pathways that might modulate the interrelationship between CAD and LV diastolic dysfunction.

$\mathrm{CV}$ risk stratification might be optimized by risk enhancers that provide incremental information to the 2013 ACC/AHA risk score $[12,13]$. Within this context, population studies previously reported an incremental value of markers of subclinical CV organ damage such as coronary artery calcification [27] and an aggregate biomarker score [34] beyond the Pooled Cohort Equations to predict CV events. Along these lines, echocardiographic profiles indicative of subclinical LV abnormalities seem promising for CV risk stratification given their independent predictive value for $\mathrm{CV}$ events in the community $[15,16,18]$. To our knowledge, our longitudinal population study was the first to assess the incremental value of echocardiographic profiles for $\mathrm{CV}$ disease prediction beyond an ASCVD risk score that has been clinically endorsed by professional cardiology societies. So far, previous studies only demonstrated the additive value of LV hypertrophy for CV disease prediction to population-based CV risk scores $[19,20]$. For instance, in 3980 CARDIA participants LV mass predicted future CV events independently of the Framingham Risk Score and significantly improved discrimination and reclassification of future CV disease [20].

In our outcome analyses, we demonstrated an incremental prognostic value of echocardiographic profiling beyond the 2013 ACC/AHA risk score only in subjects with an intermediate-to-high ASCVD risk. Indeed, in subjects with a 10-year ASCVD risk above 7.5\% at baseline, the risk for a CV event increased significantly if at least one LV abnormality was present. Compared to the average 
A) LV remodeling and dysfunction by history of cardiovascular disease
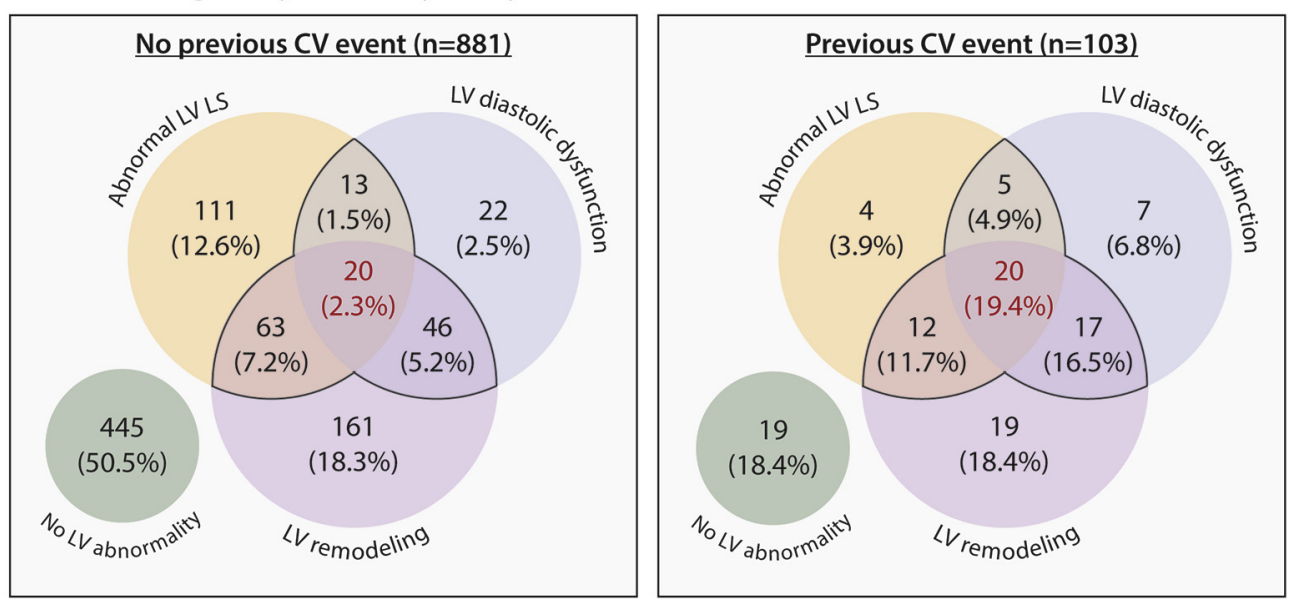

B) LV remodeling and dysfunction by 10-year ASCVD risk

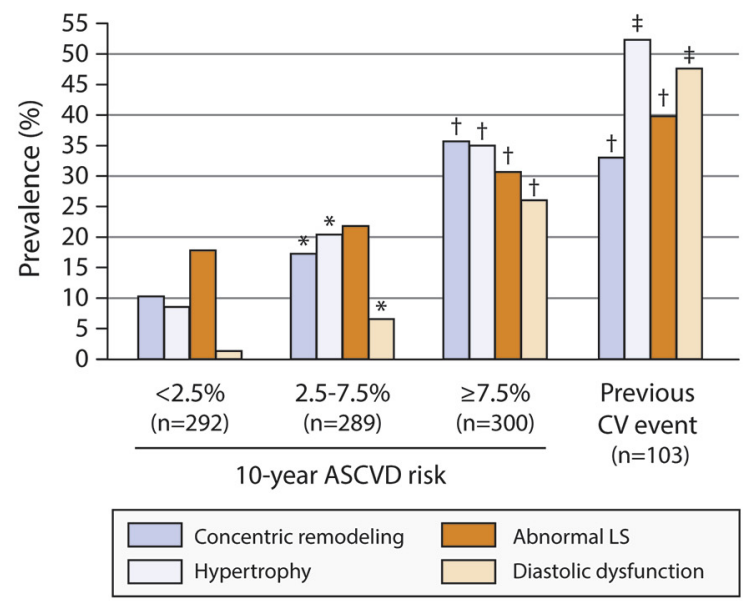

C) No. of LV abnormalities by 10-year ASCVD risk

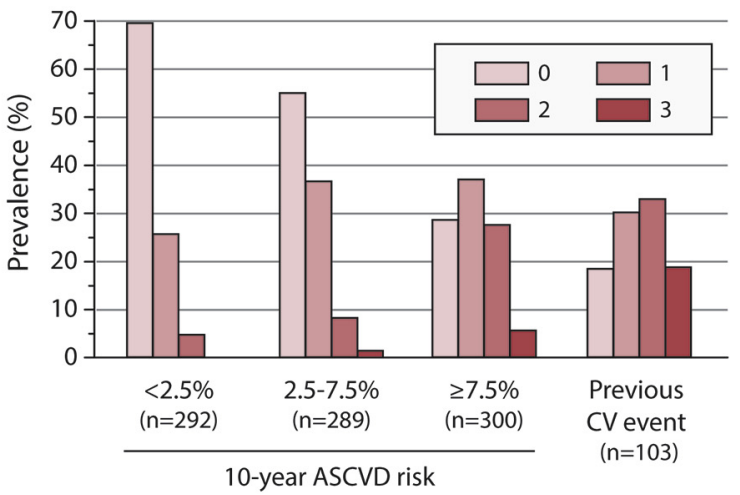

Fig. 1. Prevalence of Subclinical Left Ventricular Abnormalities by History of Cardiovascular Disease (panel A) and 10-year ASCVD Risk (panels B and C). ${ }^{\text {a }}<<0.05$ versus $<2.5 \%$ 10 -year ASCVD risk; ${ }^{b} P<0.05$ versus $<7.5 \% 10$-year ASCVD risk; ${ }^{c} P<0.05$ versus all 10 -year ASCVD risk groups. Abbreviations: ASCVD, atherosclerotic cardiovascular disease; CV, cardiovascular; LS, longitudinal strain.

population risk for a CV event, the risk of having a CV event during follow-up were three-fold higher in subjects with a 10-year ASCVD risk $\geq 7.5 \%$ and $\geq 1 \mathrm{LV}$ abnormality at baseline. Particularly the addition of LV diastolic dysfunction to a ASCVD risk score-based model improved the prognostic accuracy in predicting future CV events. In contrast, echocardiographic profiling did not improve the $\mathrm{CV}$ risk prediction beyond the 2013 ACC/AHA risk score in individuals with a 10-year ASCVD risk below 7.5\%.

In contrast to laboratory testing and a 12-lead electrocardiogram, current guidelines do not support the use of cardiac imaging modalities such as echocardiography for basic screening in primary prevention [13,23]. Among other risk enhancers [12], echocardiographic profiling might nevertheless supplement the approach currently used for prediction and prevention of CV disease. Based 


\section{A) Cumulative incidence of CV events}

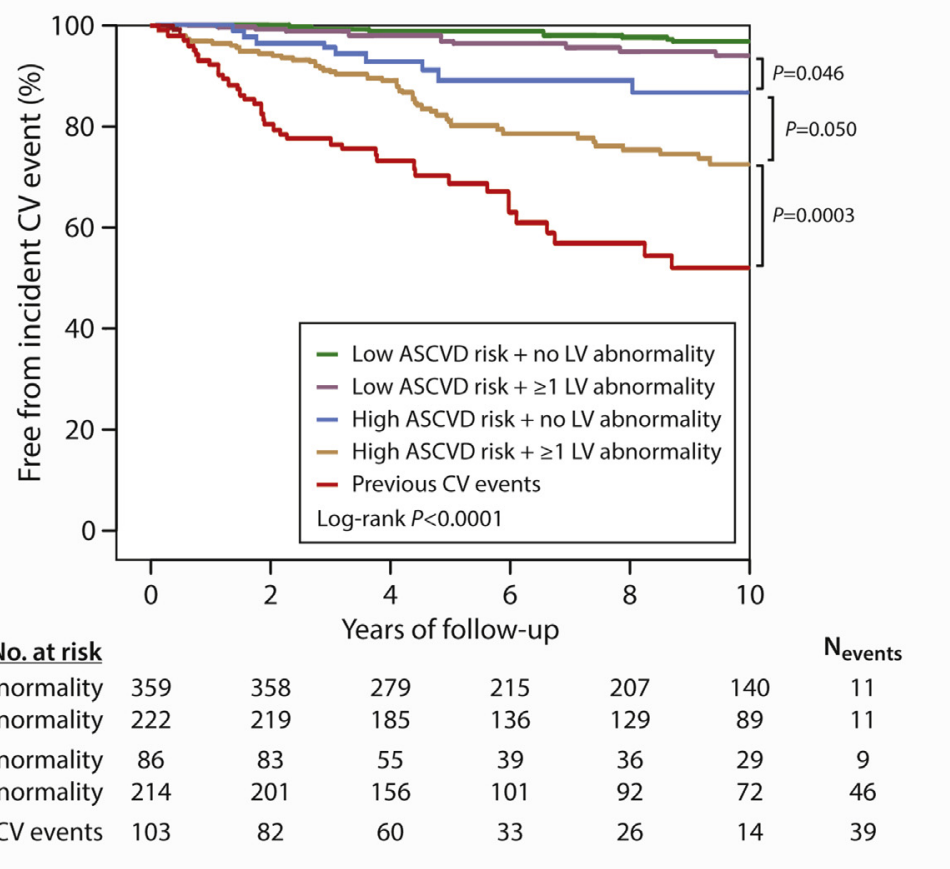

\section{B) Cox Hazard Ratios for CV events}

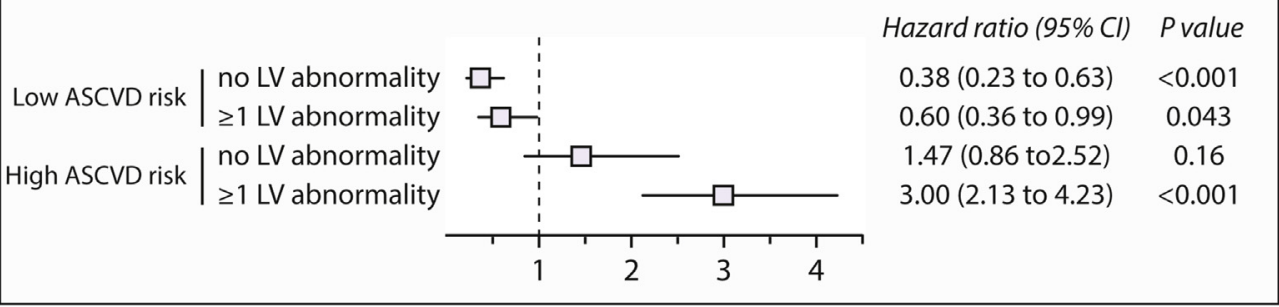

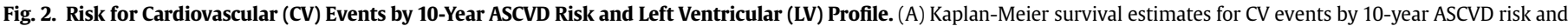

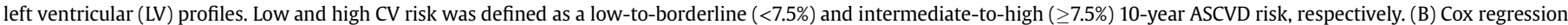

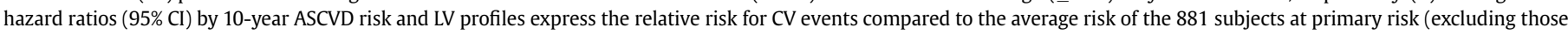
with CV events prior to the baseline examination).

Table 2

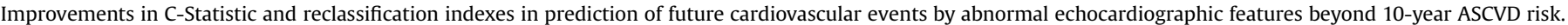

\begin{tabular}{|c|c|c|c|c|c|c|}
\hline \multicolumn{3}{|c|}{ C-statistic } & \multicolumn{2}{|c|}{$\begin{array}{l}\text { Integrated Discrimination } \\
\text { Improvement }\end{array}$} & \multicolumn{2}{|c|}{ Net Reclassification Improvement } \\
\hline Model & $\Delta$ AUC $(95 \% \mathrm{CI})$ & $P$ value & Absolute IDI (\%) & $P$ value & NRI $(95 \% \mathrm{CI})$ & $P$ value \\
\hline \multicolumn{7}{|c|}{ Low-to-borderline vs intermediate-to-high 10 -year ASCVD risk } \\
\hline + LV concentric remodeling & $0.010(-0.014$ to 0.034$)$ & 0.40 & $0.0003(0.42 \%)$ & 0.81 & $0.25(0.030$ to 0.46$)$ & 0.026 \\
\hline + LV hypertrophy & $0.024(-0.001$ to 0.049$)$ & 0.059 & $0.0038(5.87 \%)$ & 0.25 & $0.37(0.15$ to 0.60$)$ & 0.0011 \\
\hline + Abnormal LV LS & $0.027(0.001$ to 0.053$)$ & 0.040 & $0.0065(9.99 \%)$ & 0.16 & $0.31(0.087$ to 0.53$)$ & 0.0066 \\
\hline+ LV diastolic dysfunction & $0.026(0.007$ to 0.046$)$ & 0.0087 & $0.016(23.9 \%)$ & 0.016 & $0.46(0.25$ to 0.67$)$ & $<0.001$ \\
\hline$+\geq 1$ LV abnormality & $0.029(0.004$ to 0.053$)$ & 0.024 & $0.0096(14.6 \%)$ & 0.0085 & $0.54(0.33$ to 0.76$)$ & $<0.001$ \\
\hline
\end{tabular}

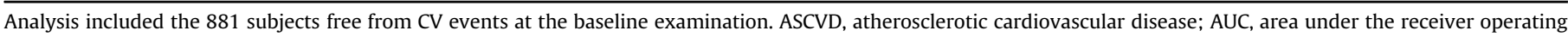
characteristic curve; LS, longitudinal strain; LV, left ventricular.

on our findings, individuals with a 10-year ASCVD risk exceeding the 7.5\% threshold might benefit from an echocardiographic screening examination. Such targeted imaging approach would enhance the cost-benefit ratio and applicability of echocardiography in risk stratification and management of CV disease.

In the future, echocardiographic findings might thus steer the discussion between clinicians and patients at substantial ASCVD risk and help decide on the type and intensity of preventive measures. Evidently, further studies should first confirm the complementarity between preselection by traditional ASCVD risk grading and echocardiographic screening for CV disease prediction. Moreover, large-scale outcome studies should evaluate the usefulness of echocardiographic profiling for guiding downstream testing and therapies in CV prevention and should, in extent, assess the 
applicability and cost-effectiveness of such echocardiographic screening.

STUDY LIMITATIONS. Our study has to be interpreted within the context of its limitations and strengths. First, echocardiographic measurements are prone to measurement errors due to signal noise, acoustic artefacts and angle dependency. However, two experienced observer recorded all echocardiographic images using a standardized imaging protocol. All echocardiographic recordings were centrally post-processed by two experienced observers with good reproducibility. Second, although the 2013 ACC/AHA risk score was not specified for heart failure (in contrast to other population-based risk scores $[19,35])$, it is the only ASCVD risk score endorsed by American cardiology societies for risk assessment in primary prevention. Third, the participation of exclusively Caucasian Europeans in our study limits the extrapolation of our findings to other ethnicities.

CONCLUSIONS. Echocardiographic profiling enhanced CV risk stratification in individuals at intermediate-to-high ASCVD risk. Future studies should investigate the clinical utility and costeffectiveness of the complementary use of traditional ASCVD risk scores and targeted echocardiographic screening for CV disease prediction and management.

PERSPECTIVES. In primary prevention, non-invasive imaging tools such as echocardiography might prove useful to better stratify the risk for future CV disease in subjects with an elevated ASCVD risk as assessed by traditional risk scoring. Our findings justify further studies to investigate the effects of targeted echocardiographic screening on CV disease prevention in individuals at moderate-to-high ASCVD risk. We advocate studies evaluating the clinical utility and cost-effectiveness of combined use of endorsed ASCVD risk scores and targeted echocardiographic screening for the prediction and prevention of CV disease.

\section{Appendix A. Supplementary data}

Supplementary data to this article can be found online at https://doi.org/10.1016/j.ijcard.2019.09.061.

\section{References}

[1] D. Mozaffarian, E.J. Benjamin, A.S. Go, D.K. Arnett, M.J. Blaha, M. Cushman, et al., Heart disease and stroke statistics-2016 update: a report from the American heart association, Circulation 133 (2016) 38-360, https://doi.org/ 10.1161/CIR.0000000000000350.

[2] A. Timmis, N. Townsend, C. Gale, R. Grobbee, N. Maniadakis, M. Flather, et al., European society of cardiology: cardiovascular disease statistics 2017, Eur. Heart J. 39 (2018) 508-579, https://doi.org/10.1093/eurheartj/ehx628.

[3] T. Kuznetsova, L. Herbots, Y. Jin, K. Stolarz-Skrzypek, J.A. Staessen, Systolic and diastolic left ventricular dysfunction: from risk factors to overt heart failure, Expert Rev. Cardiovasc Ther. 8 (2010) 251-258, https://doi.org/10.1586/ erc.10.3.

[4] W. Lieb, P. Gona, M.G. Larson, J. Aragam, M.R. Zile, S. Cheng, et al., The natural history of left ventricular geometry in the community: clinical correlates and prognostic significance of change in LV geometric pattern, JACC Cardiovasc. Imaging 7 (2014) 870-878, https://doi.org/10.1016/j.jcmg.2014.05.008.

[5] N. Cauwenberghs, J. Knez, J. D’hooge, L. Thijs, W.-Y. Yang, F.-F. Wei, et al., Longitudinal changes in LV structure and diastolic function in relation to arterial properties in general population, JACC Cardiovasc. Imaging 10 (2017) 1307-1316, https://doi.org/10.1016/j.jcmg.2016.10.018.

[6] T. Kuznetsova, E. Nijs, N. Cauwenberghs, J. Knez, L. Thijs, F. Haddad, et al., Temporal changes in left ventricular longitudinal strain in general population: clinical correlates and impact on cardiac remodeling, Echocardiography 36 (2019) 458-468, https://doi.org/10.1111/echo.14246.

[7] C.W. Yancy, M. Jessup, B. Bozkurt, J. Butler, D.E.J. Casey, M.H. Drazner, et al., ACCF/AHA guideline for the management of heart failure: a report of the American College of cardiology foundation/American heart association task force on practice guidelines, J. Am. Coll. Cardiol. 62 (2013) 147-239, https:// doi.org/10.1016/j.jacc.2013.05.019, 2013.

[8] D.C.J. Goff, D.M. Lloyd-Jones, G. Bennett, S. Coady, R.B. D’Agostino, R. Gibbons et al., ACC/AHA guideline on the assessment of cardiovascular risk: a report of the American College of cardiology/American heart association task force on practice guidelines, Circulation 129 (2013) S49-S73, https://doi.org/10.1161/ 01.cir.0000437741.48606.98, 2014

[9] M.B. Mortensen, B.G. Nordestgaard, S. Afzal, E. Falk, ACC/AHA guidelines superior to ESC/EAS guidelines for primary prevention with statins in nondiabetic Europeans: the copenhagen general population study, Eur. Heart J. 38 (2017) 586-594, https://doi.org/10.1093/eurheartj/ehw426.

[10] W.T. Qureshi, E.D. Michos, P. Flueckiger, M. Blaha, V. Sandfort, D.M. Herrington, et al., Impact of replacing the pooled cohort equation with other cardiovascular disease risk scores on atherosclerotic cardiovascular disease risk assessment (from the Multi-Ethnic Study of Atherosclerosis [MESA]), Am. J. Cardiol. 118 (2016) 691-696, https://doi.org/10.1016/ j.amjcard.2016.06.015.

[11] D.M. Lloyd-Jones, L.T. Braun, C.E. Ndumele, S.C.J. Smith, L.S. Sperling, S.S. Virani, et al., Use of risk assessment tools to guide decision-making in the primary prevention of atherosclerotic cardiovascular disease, Circulation (2018), CIR0000000000000638, https://doi.org/10.1161/ CIR.0000000000000638.

[12] S.M. Grundy, N.J. Stone, A.L. Bailey, C. Beam, K.K. Birtcher, R.S. Blumenthal, et al., AHA/ACC/AACVPR/AAPA/ABC/ACPM/ADA/AGS/APhA/ASPC/NLA/PCNA guideline on the management of blood cholesterol, Circulation (2018), https:// doi.org/10.1161/CIR.0000000000000625, 2018:CIR0000000000000625.

[13] P.K. Whelton, R.M. Carey, W.S. Aronow, D.E.J. Casey, K.J. Collins, C. Dennison Himmelfarb, et al., ACC/AHA/AAPA/ABC/ACPM/AGS/APhA/ASH/ASPC/NMA/ PCNA guideline for the prevention, detection, evaluation, and management of high blood pressure in adults: a report of the American College of cardiology/ American heart association task force on clinical Pr, Circulation 138 (2017) 484-594, https://doi.org/10.1161/CIR.0000000000000596, 2018.

[14] P. Muntner, L.D. Colantonio, M. Cushman, D.C.J. Goff, G. Howard, V.J. Howard, et al., Validation of the atherosclerotic cardiovascular disease Pooled Cohort risk equations, J. Am. Med. Assoc. 311 (2014) 1406-1415, https://doi.org/ 10.1001/jama.2014.2630.

[15] B.A. Vakili, P.M. Okin, R.B. Devereux, Prognostic implications of left ventricular hypertrophy, Am. Heart J. 141 (2001) 334-341, https://doi.org/10.1067/ mhj.2001.113218.

[16] T. Kuznetsova, N. Cauwenberghs, J. Knez, W.-Y. Yang, L. Herbots, J. D’hooge, et al., Additive prognostic value of left ventricular systolic dysfunction in a population-based cohort, Circ. Cardiovasc. Imaging 9 (2016), e004661, https:// doi.org/10.1161/CIRCIMAGING.116.004661.

[17] S. Cheng, E.L. McCabe, M.G. Larson, A.A. Merz, E. Osypiuk, B.T. Lehman, et al., Distinct aspects of left ventricular mechanical function are differentially associated with cardiovascular outcomes and all-cause mortality in the community, J. Am. Heart Assoc. 4 (2015), e002071, https://doi.org/10.1161/ JAHA.115.002071.

[18] T. Kuznetsova, L. Thijs, J. Knez, L. Herbots, Z. Zhang, J.A. Staessen, Prognostic value of left ventricular diastolic dysfunction in a general population, J. Am. Heart Assoc. 3 (2014), e000789, https://doi.org/10.1161/JAHA.114.000789.

[19] H. Chahal, D.A. Bluemke, C.O. Wu, R. McClelland, K. Liu, S.J. Shea, et al., Heart failure risk prediction in the multi-ethnic study of atherosclerosis, Heart 101 (2015) 58-64, https://doi.org/10.1136/heartinl-2014-305697.

[20] A.C. Armstrong, D.R.J. Jacobs, S.S. Gidding, L.A. Colangelo, O. Gjesdal, C.E. Lewis, et al., Framingham score and LV mass predict events in young adults: CARDIA study, Int. J. Cardiol. 172 (2014) 350-355, https://doi.org/10.1016/ j.ijcard.2014.01.003.

[21] N. Cauwenberghs, S. Ravassa, L. Thijs, F. Haddad, W.-Y. Yang, F.-F. Wei, et al., Circulating biomarkers predicting longitudinal changes in left ventricular structure and function in a general population, J. Am. Heart Assoc. 8 (2019), e010430, https://doi.org/10.1161/JAHA.118.010430.

[22] R.M. Lang, L.P. Badano, V. Mor-Avi, J. Afilalo, A. Armstrong, L. Ernande, et al., Recommendations for cardiac chamber quantification by echocardiography in adults: an update from the American society of echocardiography and the european association of cardiovascular imaging, J. Am. Soc. Echocardiogr. 28 (2015) 1-39, https://doi.org/10.1016/j.echo.2014.10.003.

[23] B. Williams, G. Mancia, W. Spiering, E. Agabiti Rosei, M. Azizi, M. Burnier, et al., 2018 ESC/ESH Guidelines for the management of arterial hypertension, Eur. Heart J. 39 (2018) 3021-3104, https://doi.org/10.1093/eurheartj/ehy339.

[24] N. Cauwenberghs, J. Knez, L. Thijs, F. Haddad, T. Vanassche, W.-Y. Yang, et al. Relation of insulin resistance to longitudinal changes in left ventricular structure and function in a general population, J. Am. Heart Assoc. 7 (2018), https://doi.org/10.1161/JAHA.117.008315.

[25] M.J. Pencina, R.B.S. D’Agostino, E.W. Steyerberg, Extensions of net reclassification improvement calculations to measure usefulness of new biomarkers, Stat. Med. 30 (2011) 11-21, https://doi.org/10.1002/sim.4085.

[26] J.-H. Park, J.H. Park, B. Ovbiagele, H.-M. Kwon, J.-S. Lim, J.Y. Kim, et al., New pooled cohort risk equations and presence of asymptomatic brain infarction,

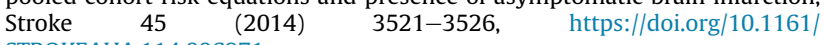
STROKEAHA.114.006971.

[27] J. Yeboah, R. Young, R.L. McClelland, J.C. Delaney, T.S. Polonsky, F.Z. Dawood, et al., Utility of nontraditional risk markers in atherosclerotic cardiovascular disease risk assessment, J. Am. Coll. Cardiol. 67 (2016) 139-147, https:// doi.org/10.1016/j.jacc.2015.10.058.

[28] Y.K. Cho, C.H. Jung, Y.M. Kang, J.Y. Hwang, E.H. Kim, D.H. Yang, et al., ACC/AHA cholesterol guideline versus 2004 NCEP ATP III guideline in the prediction of coronary artery calcification progression in a Korean population, J. Am. Heart Assoc. 5 (2016), https://doi.org/10.1161/JAHA.116.003410, 2013.

[29] V. Xanthakis, D.M. Enserro, J.M. Murabito, J.F. Polak, K.C. Wollert, J.L. Januzzi, et al., Ideal cardiovascular health: associations with biomarkers and 
subclinical disease and impact on incidence of cardiovascular disease in the Framingham offspring study, Circulation 130 (2014) 1676-1683, https:// doi.org/10.1161/CIRCULATIONAHA.114.009273.

[30] G. Tocci, I. Figliuzzi, V. Presta, N. Attalla El Halabieh, B. Citoni, R. Coluccia, et al., Adding markers of organ damage to risk score models improves cardiovascular risk assessment: prospective analysis of a large cohort of adult outpatients, Int. J. Cardiol. 248 (2017) 342-348, https://doi.org/10.1016/ j.ijcard.2017.07.078.

[31] T. Ohara, W.C. Little, Evolving focus on diastolic dysfunction in patients with coronary artery disease, Curr. Opin. Cardiol. 25 (2010) 613-621, https:// doi.org/10.1097/HCO.0b013e32833f0438.

[32] M.M. Redfield, S.J. Jacobsen, J.C. Burnett, D.W. Mahoney, K.R. Bailey, R.J. Rodeheffer, Burden of systolic and diastolic ventricular dysfunction in the community: appreciating the scope of the heart failure epidemic, J. Am. Med.
Assoc. 289 (2003) 194-202, https://doi.org/10.1016/S1062-1458(03)00178-8.

[33] T. Fatima, S. Hashmi, A. Iqbal, A.J. Siddiqui, S.A. Sami, N. Basir, et al., Untargeted metabolomic analysis of coronary artery disease patients with diastolic dysfunction show disturbed oxidative pathway, Metabolomics 15 (2019) 98, https://doi.org/10.1007/s11306-019-1559-5.

[34] E. Akintoye, A. Briasoulis, L. Afonso, Biochemical risk markers and 10-year incidence of atherosclerotic cardiovascular disease: independent predictors, improvement in pooled cohort equation, and risk reclassification, Am. Heart J. 193 (2017) 95-103, https://doi.org/10.1016/j.ahj.2017.08.002.

[35] S.K. Agarwal, L.E. Chambless, C.M. Ballantyne, B. Astor, A.G. Bertoni, P.P. Chang et al., Prediction of incident heart failure in general practice: the Atherosclerosis Risk in Communities (ARIC) Study, Circ. Heart Fail. 5 (2012) 422-429, https://doi.org/10.1161/CIRCHEARTFAILURE.111.964841. 